\title{
ALTERNATIVAS BIOLÓGICAS PARA EL CONTROL DE Fusarium oxysporum EN ARVEJA EN PAMPLONA, NORTE DE SANTANDER
}

\author{
BIOLOGICAL ALTERNATIVES FOR THE CONTROL OF Fusarium \\ oxysporum IN PEA CROP IN PAMPLONA, NORTH OF SANTANDER
}

\section{${ }^{1}$ Leónides Castellanos González, ${ }^{2}$ Joe Manuel Torrado Martínez, ${ }^{3}$ Nestor Céspedes Novoa}

\author{
${ }^{1}$ PhD. en Ciencias Agrícolas. Universidad de Pamplona. Colombia \\ 2 Ingeniero Agrónomo. Universidad de Pamplona, Colombia \\ ${ }^{3}$ Administrador Ambiental y de los Recursos Naturales. ASPAGRO, Pamplona, Colombia \\ ${ }^{1}$ leonides.castellanos@unipamplona.edu.co, ${ }^{2}$ joe.torrado@hotmail.com, \\ ${ }^{3}$ nestorcespedeshse@gmail.com
}

\section{RESUMEN}

Contextualización: la marchitez por Fusarium es la enfermedad más importante de la arveja (Pisum sativum) en Pamplona, donde se hace un uso indiscriminado de químicos

Vacío de conocimiento: existe poca evidencia científica sobre el uso y la eficacia de biocontroladores para la marchitez de la arveja por Fusarium en este municipio.

Propósito del estudio: el objetivo de la investigación fue evaluar alternativas biológicas de manejo para la marchitez por Fusarium spp. en el cultivo de arveja, en Pamplona, Norte de Santander con el fin de dar recomendaciones a los agricultores sobre su posible uso y proyectar investigaciones futuras.
Metodología: se condujo una investigación experimental, donde se evaluaron seis tratamientos: tres con biopreparados producidos por la Asociación de Productores Agropecuarios (ASPAGRO), solos o combinados, un antagonista comercial (Trichoderma harzianum), un tratamiento químico con benomil, y un testigo. Se identificó al Fusarium oxysporum como causante de la marchitez de la arveja en Pamplona. Se verificó que el producto comercial a base de Trichoderma harzianum logra reducir la incidencia y el Área Bajo la Curva de Progreso de la Enfermedad (ABCPE) de la marchitez por Fusarium en arveja de forma similar al Benomil. 
Resultados y Conclusiones: el análisis de varianza para la incidencia de la marchitez demostró diferencia entre los tratamientos, los momentos de evaluación y entre las interacciones de los tratamientos y los momentos de evaluación, así como entre los tratamientos para la incidencia y el ABCPE al final del ciclo del cultivo. Los biopreparados Caldo Rizósfera y Microorganismos de Montaña de ASPAGRO redujeron la incidencia de la enfermedad y el ABCPE con respecto al testigo. Los tres biopreparados reducen la incidencia de la marchitez, aunque los rendimientos pueden disminuir en valores próximos al 10, 25 y 30\% en los tratamientos con Trichoderma, Caldo Rizósfera y Microorganismos de montaña, respectivamente, en relación con el benomil. Por lo tanto, las dos primeras alternativas biológicas se consideran las mejores opciones para el control de la marchitez por Fusarium en arveja en Pamplona, aunque deben realizarse investigaciones para validarlos en áreas mayores.

Palabras clave: Pisum sativum; Trichoderma; Microrganismos de montaña; Caldo Rizósfera; marchitez vascular

\section{ABSTRACT}

Contextualization: Fusarium wilt is the most important pea (Pisum sativum L.) disease in Pamplona, where indiscriminate use of chemicals is applied.

Knowledge gap: There are no scientific reports of application or efficacy of biocontrollers for Fusarium wilt in pea in this municipality.

Purpose: This research aimed to evaluate biological alternatives for the control of Fusarium wilt in pea cultivation in Pamplona, Norte de Santander in order to give recommendations to farmers about its possible use and to project future research.
Methodology: An experimental design was carried out where six treatments were evaluated: three with biopreparations commercialized by the Association of Agricultural Producers (ASPAGRO), separately and in combination, a commercial antagonist (Trichoderma harzianum), a chemical treatment with benomyl, and a negative control. Fusarium oxysporum was identified as the causal agent of pea wilt in Pamplona. The commercial product based on Trichoderma harzianum was shown to reduce the incidence of the plant disease and the Area Under the Curve of Disease Progress (AUCPD) of Fusarium wilt in pea was similar to Benomil.

Results and Conclusions: The analysis of variance for the incidence of wilt showed a difference between the treatments, the evaluation moments and between the inte-ractions of the treatments and the evaluation moments, as well as between treatments for the incidence and the AUCPD at the end of the crop cycle. Compared to negative control, ASPAGRO's biopreparations of Caldo Rizósfe- ra and Microorganismos de Montaña reduced the incidence of the disease and the AUCPD. The three biopreparations achieved to reduce the incidence of wilt. Nevertheless, the yield decreased by values close to 10,25 , and $30 \%$ in the treatments with Trichoderma, Caldo Rizósfera, and Microorganismos de Montaña, respectively, in comparison of benomyl. Therefore, the first two biological means are considered the best options for the control of Fusarium wilt in peas in Pamplona, howe-ver research should be carried out to validate them in larger areas.

Keywords: Pisum sativum; Trichoderma; Microorganismos de montaña; Caldo Rizófera; vascular wilt 


\section{RESUMEN GRÁFICO}

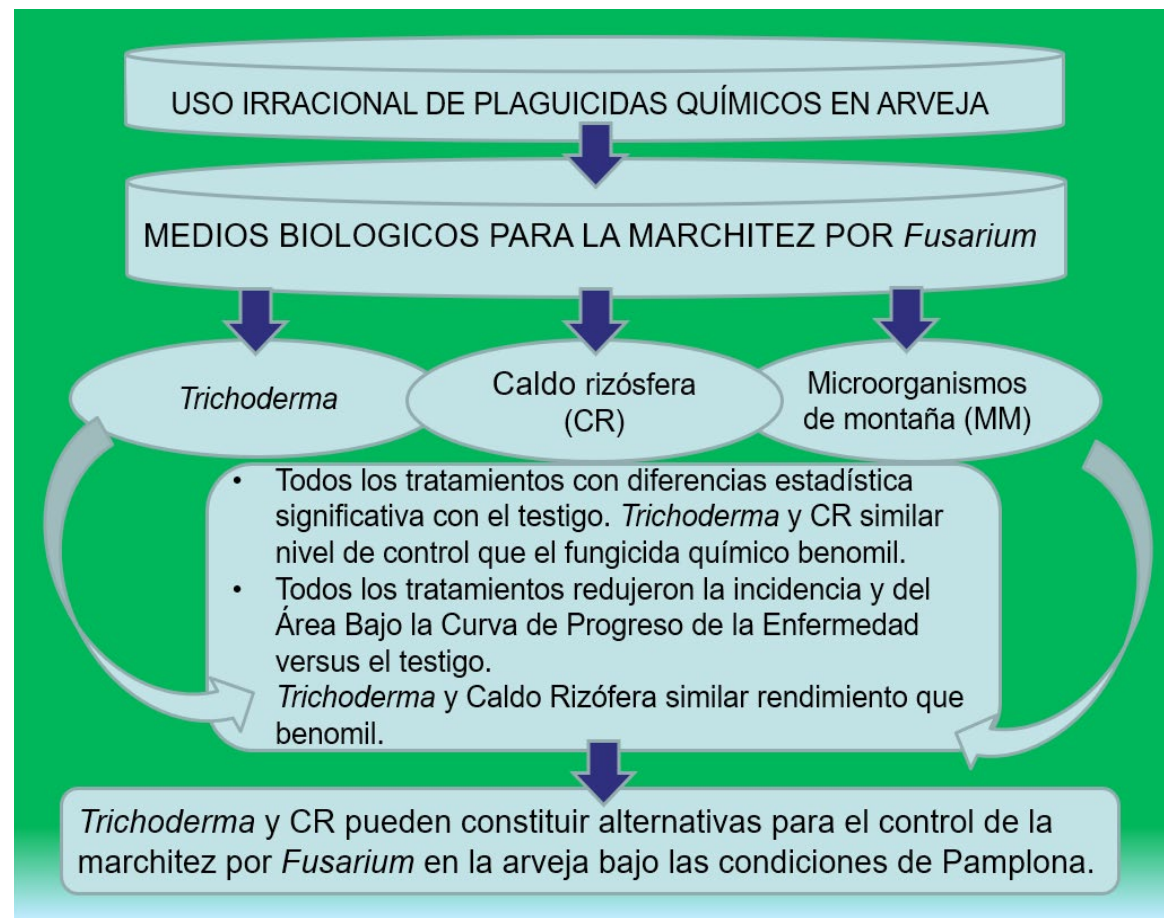

\section{INTRODUCCIÓN}

En Colombia, la pudrición de las raíces en los cultivos de arveja (Pisum sativum L.) es muy común en Nariño, Cundinamarca, Boyacá y Antioquia. Esta se presenta en las primeras fases de desarrollo del cultivo (pre y pos emergencia). Las pérdidas pueden oscilar entre $50 \%$ y $100 \%$, si no se toman medidas preventivas. La enfermedad puede causar pudriciones acuosas de las semillas o muerte prematura de estas (Tamayo, 2000; Buitrago et al. 2006). Osorio et al, (2012) caracterizaron el agente causal de la marchitez de la arveja en el municipio Manizales, y concluyeron, por métodos morfológicos, que el hongo que estaba presente causando la enfermedad era Fusarium oxysporum f.sp. pisi (CJJ Hall) WC Snyder y HN Hansen (Osorio y Castaño, 2011); sin embargo, se han relacionado con dicha enfermedad los hongos patógenos $F$. oxysporum f. sp. pisi, Fusarium solani (Mart.) Appel y Wr. f. sp. pisi, Rhizoctonia sp., y Pythium sp. (Tamayo, 2000).
Respecto al control químico, Pabón y Castaño (2012) concluyeron que el fungicida benomil era efectivo para manejar diferentes especies de Fusarium en arveja y recomiendan emplear solo las cantidades necesarias y restringir su uso a periodos críticos, para evitar la aparición de poblaciones resistentes del hongo, al igual que el uso del hongo biocontrolador Trichoderma, (Trichoplant $\AA$ WP); sin embargo, señalan que su efectividad debe mejorase con un manejo integrado de la enfermedad. Eraso et al. (2014) también estudiaron el efecto de 20 cepas de Trichoderma aisladas de diferentes zonas de Nariño, verificando que tres cepas presentaban mejor respuesta en el control de F. oxysporum f. sp. pisi. en plantas de arveja, específicamente en porcentaje de incidencia, altura de planta, longitud y materia seca de raíces. Sin embargo, en condiciones de campo, solo dos cepas manifestaron resultados significativos en el control de la enfermedad y los componentes de rendimiento. El control de 
esta enfermedad no es un problema resuelto $y$, aunque existen posibilidades de manejar la enfermedad con alternativas biológicas más amigables con el medio ambiente, la obtención de una alta eficacia en campo, con reducción de las pérdidas de cosecha, constituye un desafío para la comunidad científica (Verma et al., 2016).

Por otra parte, los Microorganismos Eficientes (ME) son una combinación de microorganismos beneficiosos de cuatro grupos principales: bacterias fototróficas, levaduras, bacterias productoras de ácido láctico y hongos de fermentación obtenidos de ecosistemas vírgenes, que tienen efecto contra bacterias $y$ hongos fitopatógenos, y actúan como fertilizante foliar y como mejoradores de la fertilidad del suelo por fijar nitrógeno atmosférico y solubilizar el fosforo (BID, 2009). Mientras que los Microorganismos de Montaña (MM) son un tipo de $M E$, o sea, que es un consorcio microbiano que se obtiene de la rizosfera de las plantas y producen los mismos beneficios que los ME (Castro-Barquero et al., 2015). Por otra parte, el Caldo Rizósfera es otro bioproducto obtenido por vía fermentativa a partir de las raíces de algunas plantas con adición de yogurt y otros productos naturales como la roca fosfórica, que incrementa microrganismos beneficiosos y se recomienda con los mismos fines que los microrganismos eficientes (Viteri et al., 2008).

En una investigación desarrollada sobre la arveja en la Vereda Monteadentro, Pamplona, Norte de Santander se recomendó el empleo de productos naturistas para el control de enfermedades con vistas a disminuir el alto uso de insumos agroquímicos (Peñaranda y Molina, 2011), lo que ha incentivado las investigaciones con alternativas biológicas. Por otra parte, se comercializan bioproductos por varias casas comerciales en Colombia y La Asociación de Productores Agropecuarios de Pamplona (ASPAGRO) produce y comercializa algunos bioproductos artesanales a base de microrganismos eficientes que podrían ayudar a disminuir el empleo de fungicidas químicos en el cultivo de la arveja; sin embargo, no se han realizado investigaciones científicas que verifiquen su eficacia en este cultivo, tanto de los comerciales, como de los artesanales, que permitan someterlo a la licencia ICA.

Considerando estos antecedentes, el objetivo de la presente investigación fue evaluar alternativas biológicas para el control de la marchitez por Fusarium en el cultivo de arveja (Pisum sativum) incluyendo biopreparados de producción local en las condiciones de Pamplona, Norte de Santander.

\section{MATERIALES Y MÉTODOS Lugar de estudio y condiciones de suelo}

La investigación se desarrolló en los cultivos de arveja de la Vereda Monteadentro del Municipio Pamplona, en la finca La Cruz de esta vereda, a una altura de 2350 m.s.n.m., y en los laboratorios de microbiología y del Centro de Investigación de Sanidad Vegetal e Bioinsumos (CISVEB) de la Universidad de Pamplona, en el periodo comprendido de marzo a diciembre de 2017. La fase de campo se desarrolló desde marzo de 2017 hasta agosto de 2017, donde concurrieron las siguientes condiciones meteorológicas de temperatura, humedad relativa y lluvia (Figura 1). 

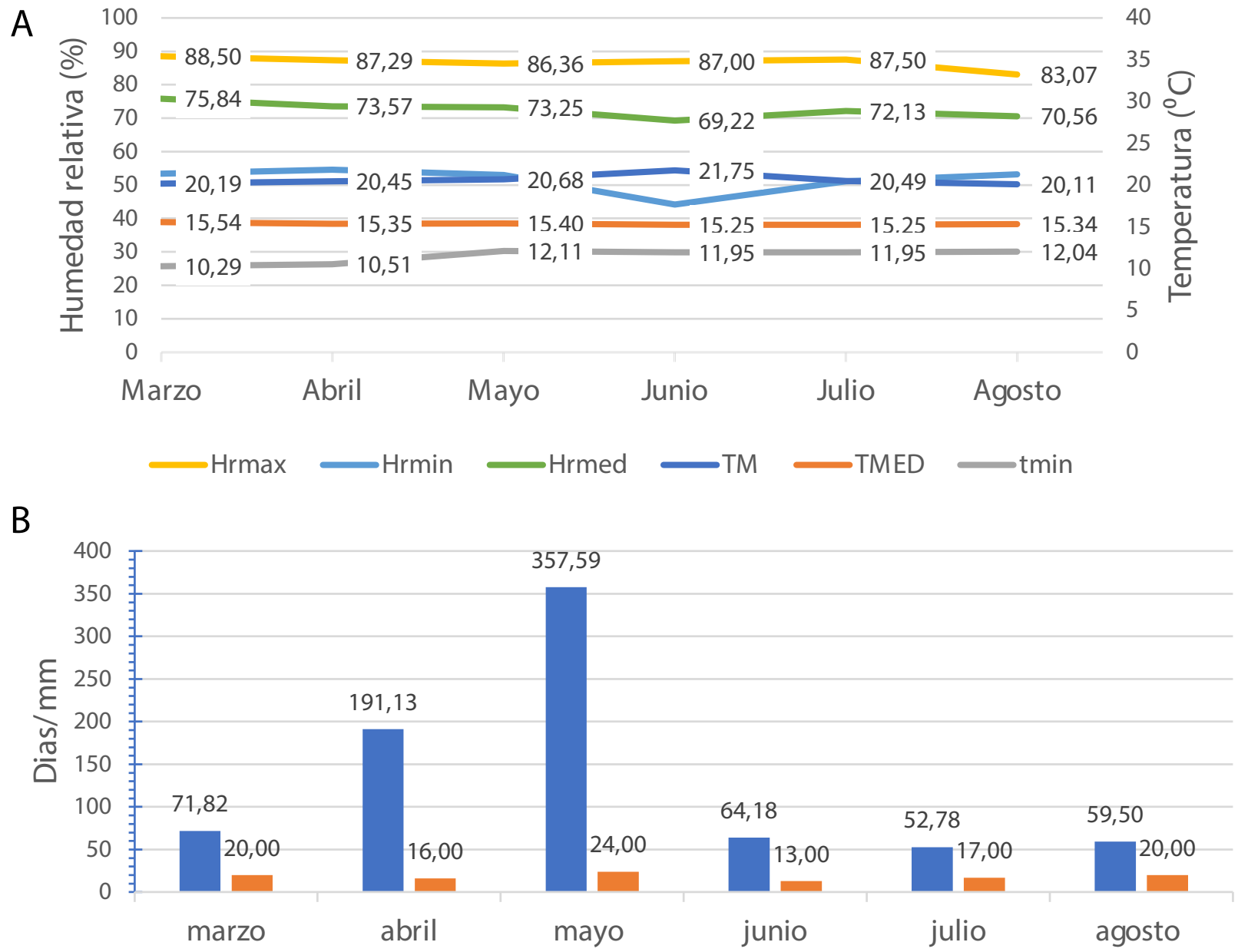

Meses 2017

T. lluvia D. Iluviosos

Figura 1. Condiciones meteorológicas que concurrieron durante el experimento de campo.

A: Humedad relativa y temperatura mensual y B: precipitación mensual

Fuente: Autores

Aplicación de alternativas y diseño de experimentos

Se llevó a cabo una investigación experimental en la Vereda Monteadentro, del municipio Pamplona, Norte de Santander, teniendo en cuenta que esta enfermedad presenta alta incidencia en la zona.

Se evaluaron seis tratamientos: tres con dos biopreparados de ASPAGRO solos o combinados, uno biológico con un biopreparado comercial de Trichoderma harzianum TRICHOX WP $5 \times 10^{8} \mathrm{ufc} / \mathrm{g}$ de la firma BIOX, un tratamiento químico con benomil (Benomyl 50 WP) de la firma Del Monte y un testigo sin tratamiento. Los ingredientes, concentración y uso propuesto de los biopreparados de ASPAGRO aparecen en la Tabla 1. 
Tabla 1. Ingredientes, concentración y uso propuesto de los biopreparados.

\begin{tabular}{|c|c|c|c|}
\hline $\begin{array}{l}\text { Biopreparados } \\
\text { artesanales }\end{array}$ & Ingredientes & $\begin{array}{l}\text { Concentración de } \\
\text { microorganismos }\end{array}$ & Uso propuesto \\
\hline $\begin{array}{l}\text { Microorganismos } \\
\text { de Montaña }\end{array}$ & $\begin{array}{l}\text { Microrganismos Eficientes, melaza y salvado } \\
\text { de arroz (Oriza sativa L.) }\end{array}$ & $1,72 \times 10^{6} \mathrm{ufc} / \mathrm{mL}$ & $\begin{array}{l}\text { Biofertilizante } \\
\text { con Bacterias } \\
\text { Solubilizadoras } \\
\text { de Fósforo y } \\
\text { antagonista }\end{array}$ \\
\hline Caldo rizosfera & $\begin{array}{l}\text { Raíces de plantas: ortiga (Urtica dioica } \\
\text { L.), borraja (Borago officinalis L.), kikuyo } \\
\text { (Pennisetum clandestinum Hochts ex } \\
\text { Chiov) trébol blanco (Trifolium repens L.) } \\
\text { conseguidas en la granja, yogurt, melaza, } \\
\text { agua oxigenada y harina de frijol (Phaseolus } \\
\text { vulgaris L.) }\end{array}$ & $1,24 \times 10^{6} \mathrm{ufc} / \mathrm{mL}$ & $\begin{array}{l}\text { Biofertilizante } \\
\text { con Bacterias } \\
\text { Solubilizadoras } \\
\text { de Fósforo y } \\
\text { antagonista }\end{array}$ \\
\hline
\end{tabular}

Fuente: Autores

Los tratamientos se realizaron cada 14 días dirigidos al suelo y a la parte inferior de las plantas en horas de la tarde. Estos se hicieron con una bomba manual de espalda de 20
L litros de capacidad. Las dosis de aplicación de cada tratamiento se relacionan en la tabla 2, siempre aplicados a $400 \mathrm{~L} / \mathrm{ha}$

Tabla 2. Dosis de aplicación y solución final.

\begin{tabular}{l|l} 
Producto & Dosis \\
\hline M. de Montaña (MM) & $1 \mathrm{~L} / 20 \mathrm{~L}$ \\
\hline Caldo Rizósfera & $1 \mathrm{~L} / 20 \mathrm{~L}$ \\
\hline MM + Caldo Rizósfera & $1 \mathrm{~L} / 20 \mathrm{~L}+1 \mathrm{~L} / 20 \mathrm{~L}$ \\
\hline Trichoderma & $0,8 \mathrm{~g} / 20 \mathrm{~L}$ \\
\hline Químico benomil & $0,5 \mathrm{~g} / 20 \mathrm{~L}(250-500 \mathrm{~g} / \mathrm{ha})$ \\
\hline
\end{tabular}

Fuente: Autores

Para el montaje del experimento, se contó con un área de $144 \mathrm{~m}^{2}(15 \times 9,6 \mathrm{~m})$, en un área baja de la finca La Cruz donde se había observado alta incidencia de marchitez típica causada por Fusarium en un cultivo de arveja existente anteriormente, es decir, había una fuente de inóculo natural.

Se realizaron labores de preparación del suelo y se programó una fertilización antes de la siembra incorporando $1 \mathrm{~kg}$ de urea, $1 \mathrm{~kg}$ de cloruro de potasio y $5 \mathrm{~kg}$ de 10-30-10.

Se empleó la variedad "Rabo Gallo", sembrada directamente en el campo en surcos separados entre sí, a una distancia de $120 \mathrm{~cm}$ y $2 \mathrm{~cm}$ entre surcos y plantas, respectivamente. El sistema de tutorado se estableció 20 días después de la siembra (dds) y un segundo tutor se colocó cuando las plantas tenían una altura promedio de $30 \mathrm{~cm}$. 
Se empleó un diseño experimental de bloques al azar con cuatro réplicas con un arreglo $6 \times 4$ (6 tratamientos con 4 réplicas), es decir, 24 unidades experimentales (parcelas). El tamaño de cada parcela experimental fue de 5 metros de largo por 4 metros de ancho con un área de $20 \mathrm{~m}^{2}$. La distancia entre plantas fue de $2 \mathrm{~cm}$ y de $1 \mathrm{~m}$ entre surcos.

\section{Incidencia de Fusarium spp.}

Para confirmar el agente causal de la enfermedad, se tomaron plantas con los síntomas que fueron trasladadas a los laboratorios de microbiología y del Centro de Investigación de Sanidad Vegetal de Bioinsumos (CISVEB) de la Universidad de Pamplona, donde se desinfestaron con $\mathrm{NaClO}_{3}$ al $5 \%$ por 2 minutos y posteriormente se incubaron en cámara húmeda y se realizaron siembras en el medio de cultivo artificial PDA (Papa Dextrosa-Agar), utilizado como medio general para el crecimiento de hongos. La identificación de los microorganismos presentes se realizó haciendo aislamientos en PDA, con la ayuda del microscopio óptico y la clave de para las especies de Fusarium (Leslie y Summerell, 2006). Se tuvo en cuenta la coloración de colonias, la utilización de la clave taxonómica con base a las estructuras reproductivas como conidios, mesoconidios, macroconidios, clamidosporas.

Para determinar la incidencia de Fusarium y el comportamiento de los tratamientos en el control de la enfermedad, se tomaron 20 plantas al azar por cada una de las parcelas, en los surcos centrados, tres días después de cada aplicación, evaluándose las plantas enfermas por Fusarium sobre el total evaluadas, cada 14 días. Con esta información se calculó la incidencia mediante la siguiente fórmula:

$\%$ de incidencia $=\frac{n(\text { plantas afectadas })}{N(\text { evaluadas })} X 100$

(Ecuación 1) (Agrios, 2005)
Se determinó el Área Bajo la Curva de Progreso de la Enfermedad (ABCPE) en cada parcela según el método de Campbell y Madden (1990), para lo cual se utilizó la siguiente fórmula:

$$
\text { ABCPE }=\Sigma\left[\left(\mathrm{X}_{\mathrm{i}}+\mathrm{X}_{\mathrm{i}}+1\right) / 2\right] *\left(\mathrm{~T}_{\mathrm{i}}+1-\mathrm{Ti}\right)
$$

(Ecuación 2) (Campbell y Madden, 1990)

\section{Donde:}

$X_{i}=$ distribución o intensidad de la enfermedad en el muestreo $\mathrm{i}$

$\mathrm{X}_{\mathrm{i}+1}=$ distribución o intensidad de la enfermedad en el muestreo i+1

$\mathrm{T}_{\mathrm{i}}=$ tiempo $_{\mathrm{i}} \mathrm{T}_{\mathrm{i}+1}=$ tiempo $_{\mathrm{i}+1}$

\section{Rendimiento del cultivo}

Al final del experimento se evaluaron indicadores del componente de rendimiento y el rendimiento de cada parcela. Los componentes del rendimiento evaluados fueron: vainas por planta en la cosecha (en 20 plantas por parcela), granos por vaina (100 vainas por parcela) y peso de las vainas. Para esta última variable, se pesaron 100 vainas por parcela para sacar el promedio. Se estimó el peso de la producción por parcela a partir del peso promedio de las vainas el número de vaina por parcela y el número de plantas por parcela. A partir de la producción y el área, se estimó el rendimiento en $t$. Se compararon los rendimientos en porcentajes contra el testigo.

\section{Análisis estadístico}

Se realizó un análisis de varianza del porcentaje de incidencia de la marchitez por Fusarium en el que se consideraron dos factores: primero, los tratamientos con seis niveles (los cinco tratamientos y el testigo); y segundo, el momento de evaluación con cuatro niveles (evaluaciones 08/07, 22/07,05/08 y 19/08). 
También se realizó un análisis de varianza de clasificación simple para el ABCPE. Previamente se verificó el cumplimiento del supuesto de normalidad de cada variable dependiente por la prueba de Shapiro-Wilks, para cada grupo, con $n=16$ y observando $p>0,05$. Se compararon las medias por la prueba de Tukey, con un $5 \%$ probabilidad de error. Se empleó en todos Ios análisis el paquete estadístico AgroEstat (Barbosa y Maldonado, 2015).

Los datos de temperaturas, humedad relativa y precipitación obtenidos en el CISVEB se procesaron en Microsoft Excel obteniéndose los gráficos de los valores mínimos, medios y máximos mensuales de la temperatura y la humedad relativa, así como de precipitación total y de días lluviosos.

\section{RESULTADOS Y DISCUSIÓN}

Los síntomas de la marchitez por Fusarium se detectaron en el tercer muestreo del experimento. A partir de ese momento, la incidencia de la enfermedad se mantuvo en incremento creciente, sobre todo en el testigo. El agente causal correspondió con Fusarium oxysporum tanto por las características de la colonia en PDA como la presencia de macroconidios típicos, mesoconidios y midroconidios (Figura 2).

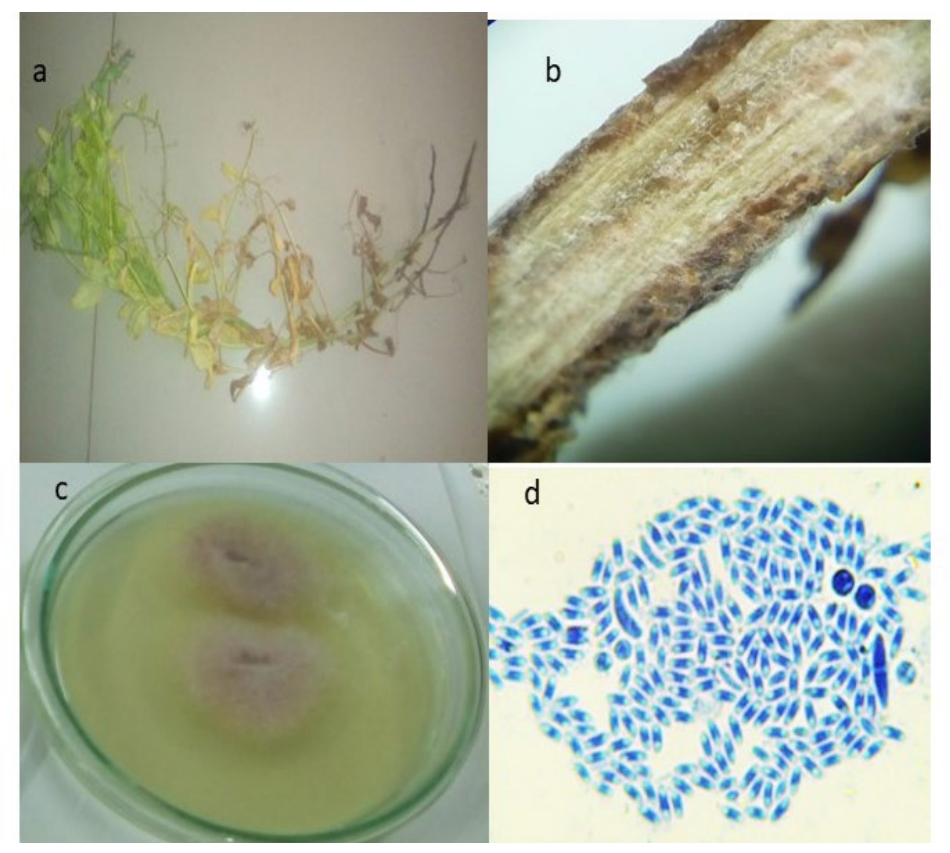

Figura 2. Planta de arveja con síntomas de amarillamiento y marchitez por Fusarium oxyporum. a); síntomas de necrosis en el tallo b); colonia de Fusarium oxyporum. en PDA c); microconidios, mesoconidios, macroconidios y clamidosporas de Fusarium oxyporum d)

Fuente: Autores

Los síntomas de la enfermedad y las características del microoorganismo aislado conidióforo, fiálides y conidios (Tabla 3) corresponden con las descripciones de Gutiérrez y
Zapata (2011) en el cultivo de arveja, y confirmado el hongo como Fusarium oxysporum según Leslie y Summerell (2006). 
Tabla 3. Características morfológicas del microrganismo aislado que permitieron identificarlo como Fusarium oxysporum

\begin{tabular}{l|l}
\multicolumn{1}{l}{ Estructuras } & \multicolumn{1}{c}{ Características } \\
Micelio & $\begin{array}{l}\text { De crecimiento rápido, algodonoso, de color blanco, pero que a medida que } \\
\text { crecía pigmentaba el medio PDA con una coloración violeta, más fácilmente } \\
\text { evidente en el reverso de las cajas de Petri. Cuando pasaba el tiempo tomaba } \\
\text { una coloración violeta pálido }\end{array}$ \\
\hline Conidios & $\begin{array}{l}\text { Presencia de microconidios, mesoconidios y macroconidios solitarios, estos } \\
\text { últimos con hasta tres septos, rectos o ligeramente curvos. }\end{array}$ \\
\hline Conidióforos & Terminando en fiálides, en ocasiones con aglomeraciones de microconidios \\
\hline Clamidosporas & $\begin{array}{l}\text { Presencia de clamidiosporas, hialinas, lisas o ligeramente rugosas, terminales o } \\
\text { intercalares en las hifas }\end{array}$ \\
\hline
\end{tabular}

Fuente: Autores

Dada la importancia de la arveja en la zona, y la marchitez por $F$. oxysporum, se debe profundizar en la identificación molecular del patógeno asilado; es decir, realizar el "DNA barcoding" del hongo, para dejar definida y depositada la secuencia genética de esta cepa, y para continuar en la búsqueda de otras especies de Fusarium que también se hayan asociado a enfermedades radiculares de la arveja en otras zonas (Tamayo, 2000).

El resultado del ANOVA realizado a la incidencia de la marchitez por Fusarium mostró valores de $F$ (Fisher) significativos $P<0,05$ para el factor Tratamientos, para el Factor Momentos de evaluación y para la interacción Tratamientos $x$ Momentos de evaluación. El mayor nivel de incidencia de la enfermedad se observó para el testigo (76,62\%) el 19/08 con diferencia estadística significativa con el resto de los tratamientos con bioproductos y con el fungicida benomil en ese momento. También presentó diferencia estadística significativa con el testigo en la evaluación anterior del 05-08.

Se observó una situación similar, en el 5-8, en que el testigo mostraba diferencia estadística con el resto de los tratamientos y también con el muestreo anterior. Los menores niveles de incidencia de la enfermedad se observaron a los 42 días de germinado el cultivo (08/07) en los tratamientos con MM, Caldo Rizósfera y el fungicida químico benomil, que difirieron del testigo, pero no de los tratamientos con Trichoderma y MM + Caldo Rizosfera (Tabla 4). 
Tabla 4. Resultado del ANOVA para la incidencia la marchitez por Fusarium en arveja para la interacción Tratamientos $\times$ Momentos de evaluación.

\begin{tabular}{l|c|c|c|c|c}
\multicolumn{7}{c}{ Incidencia (\%) / Fechas } \\
Factor & \multicolumn{7}{c}{ Momento de evaluación } \\
\hline Tratamientos & $08-07$ & $22-07$ & $05-08$ & $19-08$ & DMS (5\%) \\
\hline Testigo & $23,12 \mathrm{Da}$ & $38,50 \mathrm{Ca}$ & $60,93 \mathrm{Ba}$ & $76,62 \mathrm{Aa}$ & 5,31 \\
\hline MM + Caldo Rizósfera & $20,62 \mathrm{Dab}$ & $34,06 \mathrm{Cab}$ & $49,68 \mathrm{Bb}$ & $69,43 \mathrm{Ab}$ & 5,31 \\
\hline Trichoderma & $17,37 \mathrm{Dab}$ & $30,62 \mathrm{Cb}$ & $45,31 \mathrm{Bbc}$ & $59,00 \mathrm{Ac}$ & 5,31 \\
\hline MM & $16,87 \mathrm{Db}$ & $30,31 \mathrm{Cb}$ & $41,93 \mathrm{Bc}$ & $54,81 \mathrm{Acd}$ & 5,31 \\
\hline Caldo Rizósfera & $15,68 \mathrm{Db}$ & $30,12 \mathrm{Cb}$ & $41,75 \mathrm{Bc}$ & $54,68 \mathrm{Acd}$ & 5,31 \\
\hline Químico & $15,31 \mathrm{Db}$ & $29,68 \mathrm{Cb}$ & $40,81 \mathrm{Bc}$ & $50,62 \mathrm{Ad}$ & 5,31 \\
\hline DMS (5\%) & 5,91 & 5,91 & 5,91 & 5,91 & \\
\hline C.V. (\%) & & & 7,22 & \\
\hline
\end{tabular}

* Las letras mayúsculas comparan los momentos de evaluación en las filas y las minúsculas comparan los tratamientos en las columnas por la prueba de Tukey $(P \leq 0,05)$. DMS: diferencia mínima significativa.

Fuente: Autores

Estos resultados ponen de manifiesto la superioridad de los biopreparados y el fungicida químico benomil al inicio $y$ en otros momentos del experimento, independientemente del aumento progresivo de la enfermedad en el cultivo.

El resultado del ANOVA para el factor "Tratamientos" demostró que, en general, en los cuatro momentos de evaluación en las parcelas donde se aplicó Trichoderma, la incidencia de la enfermedad fue más baja comparada con el testigo y las tratadas con MM + Caldo Rizosfera; sin embargo, no reflejó diferencia estadística significativa con el benomil y Caldo Rizósfera. Los tratamientos con MM y MM +Caldo Rizósfera también tuvieron diferencia estadística significativa respecto al testigo. Estos resultados fueron similares a los del Área Bajo la Curva de Progreso de la Enfermedad donde se destacó el tratamiento con Trichoderma con el menor valor de porcentaje de incidencia, pero sin diferencia estadística significativa con el tratamiento químico y Caldo Rizosfera y de igual forma MM +Caldo Rizósfera tuvieron diferencia estadística significativa con el testigo (Tabla 5). 
Tabla 5. Incidencia y ABCPE de la marchitez por en los tratamientos del experimento para el control de esta enfermedad en el cultivo de arveja.

\begin{tabular}{l|c|c}
\hline Factor (Tratamientos) & Incidencia (\%) & ABCPE \\
\hline Testigo & $56,03 \mathrm{a}$ & $5240,81 \mathrm{a}$ \\
\hline MM + Caldo Rizósfera & $45,34 \mathrm{~b}$ & $4158,87 \mathrm{~b}$ \\
\hline Trichoderma $5 \times 10^{8} \mathrm{ufc} / \mathrm{mL}$ & $30,42 \mathrm{e}$ & $2734,37 \mathrm{~d}$ \\
\hline MM & $38,04 \mathrm{c}$ & $3539,37 \mathrm{c}$ \\
\hline Caldo Rizósfera & $34,57 \mathrm{~cd}$ & $3127,68 \mathrm{~cd}$ \\
\hline Químico & $33,53 \mathrm{de}$ & $3196,37 \mathrm{~cd}$ \\
\hline C.V. (\%) & 8,70 & 7,1 \\
\hline Error Típico* & 1,74 & 131,8
\end{tabular}

* Las medias con letras desiguales en las columnas difieren por la prueba de Tukey $(P \leq 0,05)$

Fuente: Autores

La comparación de las medias de la incidencia de la enfermedad mostró diferencia estadística significativa del último momento 19-08, con el mayor valor $(62,25 \%)$ con relación a los anteriores, y el resto también con los otros, evidenciando un aumento progresivo de la enfermedad en el tiempo y con diferencia estadística significativa entre todos los momentos de muestreo.

La acción de los biopreparados de ASPAGRO es atribuible, para MM, a los hongos, bacterias y actinomicentes que contiene este, fundamentalmente los del último grupo que están en una concentración de $1,65 \times 10^{6} \mathrm{ufc} / \mathrm{mL}$ y, en el caso de $C R$, al gran contenido de bacterias que contiene $1,06 \times 10^{6} \mathrm{ufc} / \mathrm{mL}$, aunque también están presentes hongos y actinomicetos en concentraciones de 1,06 $\times 10^{5} \mathrm{ufc} / \mathrm{mL}$ y $6,6 \times 10^{4} \mathrm{ufc} / \mathrm{mL}$, respectivamente (Castellanos et al., 2018).

Durante el ensayo, la temperatura media ambiental estuvo oscilando entre 14 y $16^{\circ} \mathrm{C}$. Se comprobó que los dos biopreparados de ASPAGRO y el de Trichoderma empleados pueden realizar su efecto supresor de Fusarium a esas bajas temperaturas, ya que existe preocupación respecto al efecto de Trichoderma contra patógenos fungosos foliares como Botrytis cinerea Pers. (Helford et al., 2000) por la demora en la germinación de los conidios del antagonista a temperaturas entre 12 y $14{ }^{\circ} \mathrm{C}$.

Con relación a los componentes del rendimiento los granos/vaina, estos presentaron variaciones de entre 8 y 9, para los tratamientos biológicos solos y el químico, pero disminuyó a 6 y 5 para la mezcla MM +CR y el testigo, respectivamente; mientras que las vainas/ plantas variaron entre 13, 75 y 16,25 para el tratamiento químico y los biológicos solos, y disminuyó a 9,5 para MM +CR y a 7,5 en el testigo. Además, la variable peso de las vainas (g/vaina) tuvo un comportamiento similar, con valores entre 12,5 y 15 (relativamente más altos) para los tratamientos biológicos solos y el químico, intermedio $(9,5)$ para $M M+C R$ y menor $(7,5)$ para el testigo (Figura 3A). Los rendimientos alcanzaron valores bajos, 1,59 t/ ha en el testigo y 2,25 t/ha en el tratamiento $\mathrm{MM}+\mathrm{CR}$, mientras que en el resto oscilaron 
entre 4,29 y 6,69 t/ha. El mayor valor relativo de rendimiento se obtuvo en el control químico $(6,69$ t/ha). MM solo alcanza el $70,51 \%$ y
CR el $78,82 \%$ de este valor. Sin embargo, el tratamiento de MM + CR solo alcanzó el 37\% (Figura 3B).

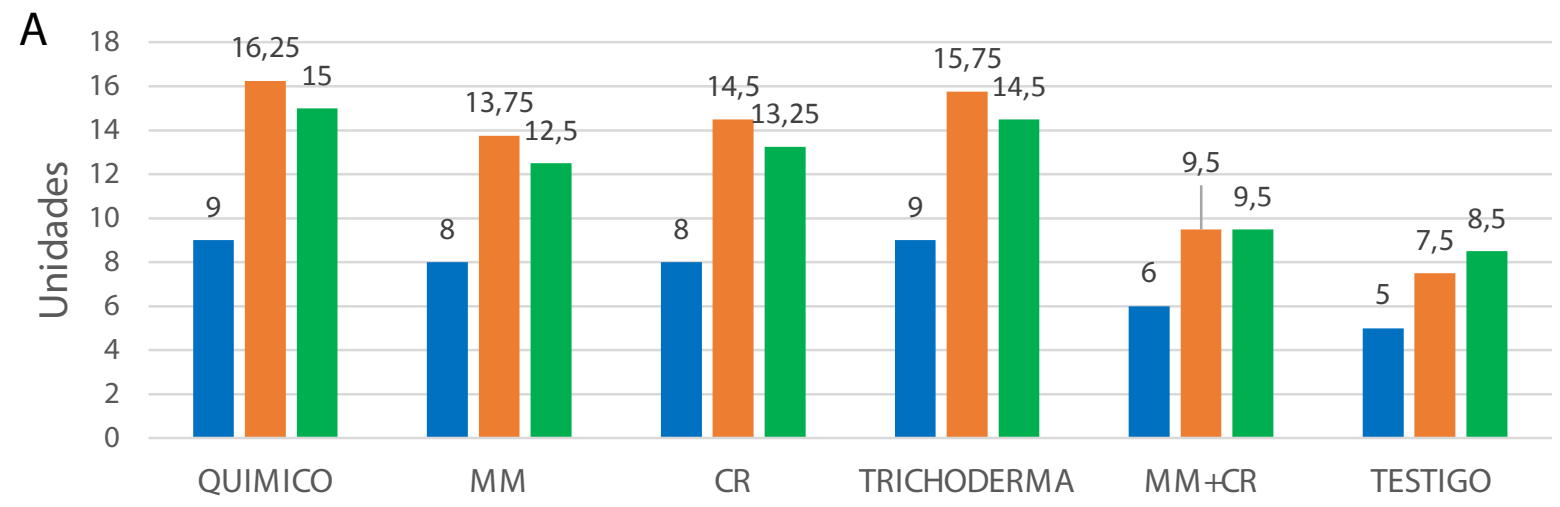

-Granos/vaina vainas/plantas gramos/vaina

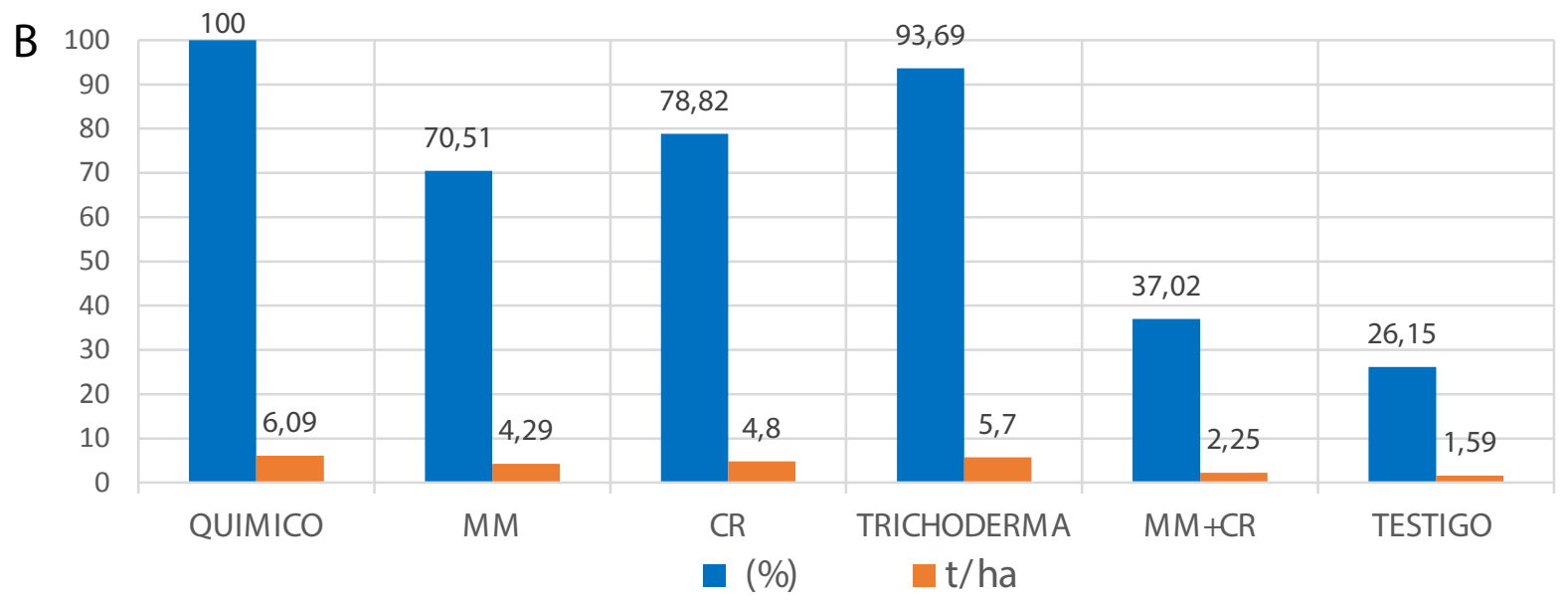

Figura 3. A. Componentes del rendimiento en los diferentes tratamientos evaluados contra la marchitez por Fusarium B. Rendimiento de arveja en los diferentes tratamientos evaluados contra la marchitez por Fusarium y porcentaje que representa respecto al químico.

Fuente: Autores

Se evidencia el efecto que hace la enfermedad sobre los componentes del rendimiento cuando no se hace un control eficaz. Se verifica la importancia la marchitez por Fusarium. en el cultivo de la arveja ya que en el testigo hubo una reducción de las tres cuartas partes del rendimiento, lo cual coincide con Eraso et al. (2014).

Los bioproductos MM y CR aplicados solos y
Trichoderma pueden constituir alternativas para el control de la marchitez por Fusarium bajo las condiciones de Pamplona, evitando el uso indiscriminado de químicos y la selección de razas resistentes a los fungicidas de alta efectividad como el benomilo, pero que actúan solo sobre un punto del metabolismo del hongo. Se hace necesario realizar validaciones de estos resultados en otros experimentos o en 
parcelas demostrativas y confirmar si los resultados de control de Caldo Rizósfera siguen superando a los de MM. En la India también se ha recomendado el uso de biocontroladores de los géneros Trichocerma, Bacillus y Pseudomonas para el control de la marchitez por Fusarium oxysporum en garbanzo (Keote et al., 2019), así como inoculantes bacterianos nativos para el control de la marchitez por Fusarium en este cultivo (Nagaraju et al., 2017 ).

La mezcla de MM y CR resultó menos eficaz que MM y CR por separado, lo que indica que, en alguno de los bioproductos, puede contener microorganismos antagónicos del otro o que no hacen sinergias entre sí. Esto se puede explicar por los diferentes orígenes y composición de los biopreparados, ya que, en el caso de MM, provenían de un consorcio que convivían en la naturaleza con un alto número de $\mathrm{ufc} / \mathrm{mL}$ de actinomicetes y $\mathrm{CR}$ con mayor $\mathrm{ucf} / \mathrm{mL}$ de otras bacterias producidas en la fermentación de las plantas y los demás sustratos (Castellanos et al., 2018).

Otros investigadores observaron que la aplicación de Trichoderma sp. mejora notablemente el crecimiento y desarrollo del cultivo de la arveja, influyendo en variables fisiológicas como germinación, área foliar, peso seco y fresco de la raíz, peso seco y fresco de la parte aérea, y longitud de raíz (Camargo y Avila, 2014). Esto se evidenció también en la presente investigación.

Resulta importante prospectar cepas nativas de este antagonista en campos de arveja de la zona, ya que, en la India, cepas nativas de Trichoderma spp. mostraron porcentajes de eficacia mayores, con diferencia significativa en relación al empleo de cepas comerciales de $T$. hamatum y $T$. viride tanto en condiciones de laboratorio como invernadero (Kripalini et al., 2019).
Constanza et al. (2010) observaron que cuatro cepas de Bacillus spp. disminuyeron la severidad de la marchitez vascular en todos los ensayos y la atribuyeron a la producción de sustancias antifúngicas que facilitaron el aumento del peso seco de las hojas de las plantas, al permitir la obtención de nutrientes. Una cepa en específico aumentó la longitud de la raíz y el tallo, por tal razón se hace necesario continuar profundizando en las especies de bacterias presentes en los dos biopreparados de ASPAGRO que se estudiaron.

En ensayos de control usando Trichoderma para los tratamientos biológicos en la variedad de arveja Santa Isabel se obtuvieron 6,50 y 7,45 granos por vaina (Eraso et al. 2014), lo cual se corresponde con los resultados del presente trabajo.

El nivel de control obtenido con los tratamientos con benomyl apoyan los resultados de Pabón y Castaño (2012) quienes obtuvieron reducción de la incidencia de $F$. oxysporum en arveja con este fungicida con relación al testigo. De igual forma estos investigadores comprobaron la reducción de la marchitez por Fusarium en este cultivo con el empleo de diferentes cepas de Trichoderma. En otros resultados, se comprobó que el carbendazin (un fungicida del grupo de los bencimidazoles muy relacionado con el benomyl) resultó ser más efectivo que otros seis fungicidas probados contra F. oxysporum (Chanu et al., 2019).

Los presentes resultados demuestran la posibilidad de emplear biocontroladores para la marchitez por Fusarium en arveja en Pamplona, en principio, con productos comerciales de T. harzianum como el que se empleó, así como los bioproductos MM y CR de ASPAGRO; sin embargo, deben validarse los resultados en áreas más extensas, debiéndose continuar las investigaciones para conseguir cepas nativas 
de Trichoderma en los cultivos de arveja, con lo que pudiera aumentar la eficacia y los efectos benéficos de este antagonista.

\section{CONCLUSIONES}

Se verifica que el biopreparado comercial a base de Trichoderma logra reducir la incidencia y el ABCPE de la marchitez por Fusarium en arveja de forma similar al benomil y no se corre riego de crear fungorresistencia de este patógeno u otros que atacan al cultivo, disminuyendo la carga tóxica en los campos.

Los biopreparados Caldo Rizósfera y del Microorganismo de Montaña de ASPAGRO pueden constituir alternativas biológicas para el manejo de la marchitez por Fusarium en arveja, ya que lograron reducir la incidencia de la enfermedad al final del ciclo del cultivo y el $A B C P E$ con respecto al testigo $y$, en el caso del primero, sin diferencia estadística con el fungicida químico.

Trichoderma comercial y los biopreparados Caldo Rizosfera de ASPAGRO y Microorganismo de Montaña, logran disminuir la incidencia la marchitez por Fusarium, aunque los rendimientos se pueden reducir en valores próximos al 10, 25 y 30\% para cada biopreparado, respectivamente, con respecto al empleo del benomil.

En general Trichoderma y Caldo Rizósfera fueron las alternativas biológicas de mejor comportamiento y pueden constituir alternativas para el control de la marchitez por Fusarium en la arveja bajo las condiciones de Pamplona.

\section{CONTRIBUCIÓN DE LA AUTORÍA}

Primer autor: elaboración del proyecto, supervisión de la ejecución de la investigación en campo y laboratorio, realización de los análisis estadísticos, redacción de la versión final del artículo. Segundo autor: elaboración del proyecto, ejecución de la investigación en campo, participación en los análisis de laboratorio, recolección de la información y procesamiento de la misma, y redacción de la versión inicial del informe. Tercer autor: participación en la elaboración del proyecto, aporte en los productos locales, asesoramiento sobre las aplicaciones de los biopreparados, participación en la revisión de todas las versiones de los informes.

\section{AGRADECIMIENTOS}

Los autores agradecen el apoyo brindado por la Asociación de Productores Agropecuarios de Pamplona (ASPAGRO) y, en particular, a la Finca Sol Vida donde se produjeron los biopreparados locales y a los agricultores de la finca La Cruz de la Vereda Monteadentro donde se realizó el experimento de campo, así como a la Universidad de Pamplona por la cooperación con los laboratorios de microbiología y la información meteorológica. 


\section{LITERATURA CITADA}

Agrios, G. (2005). Plant Pathology. Florida University. Elsevier Academic Press.

Barbosa, J., y Maldonado, J. (2015). Experimentação agronômica e AgroEstat:sistema para análises estatísticas de ensaios agronômicos. 2, Jaboticabal: Departamento de Ciências ExatasUniversidade Estadual Paulista (UNESP). https:// scholar.google.com.br/scholar?hl=pt-BR\&as_sdt $=0,5 \&$ cluster $=17992883676024259991$

BID. (2009). Banco Interamericano de Desarrollo. Manual Práctico de Uso de EM. Convenio Fondo Especial de Japón / BID ATN/JO-10792 UR. Uruguay. $35 p$.

Buitrago, J.Y., Duarte C.J., y Sarmiento, A. (2006). El cultivo de la arveja en Colombia. Federación Nacional de Cultivadores de Cereales y Leguminosas - FENALCE- y Fondo Nacional Cerealista. Ed. Produmedios, Bogotá. Colombia.

Camargo, D.F., y Ávila, E.R. (2014). Efectos de Trichoderma en el desarrollo de la arveja (Pisum sativum), Ciencia y Agricultura, 11(1), 91-100.

Castellanos, L., Céspedes, N., Sequeda, A., Jaime, J.E., y Niño, L.J. (2018). Caracterización microbiológica de cinco biopreparados artesanales. Revista Científica Agroecosistemas. 6(3), 57-65.

Castro-Barquero, L., Murillo-Roos, M., Uribe-Lorío, L., y Ymata-Chinchilla, R. (2015). Inoculación al suelo con Pseudomonas fluorescens, Azospirillum oryzae, Bacillus subtilis y Microorganismos de Montaña (MM) y su efecto sobre un sistema de rotación soya-tomate bajo condiciones de invernadero. Agronomía Costarricense, 39(3), 21-36.

Chanu, W.T., Sinha, B., Devi, S., Sharma R., y Singh, N.G. (2019). In vitro assessment of fungicides against Fusarium oxysporum $f$. sp. pisi causing Fusarium wilt of pea. Journal of Pharmacognosy and Phytochemistry, 8(4), 2487-2490. http://www.phytojournal.com/archives/2019/voI8issue4/PartAP/8-4-368-739.pdf

Constanza, L.; Sánchez, L.C., Cuervo, J., Bautista, D., González, L., y Guevara, M. (2010). Evaluación del efecto biocontrolador de Bacillus spp., frente a Fusarium spp., bajo condiciones de invernadero en Rosmarinus offcinalis L. Publicación Científica En Ciencias Biomédicas. 8(13), 1- 120.

Eraso, C., Acosta, J., Salazar, C., y Betancourth, C. (2014). Evaluación de cepas de Trichoderma spp. para el manejo del amarillamiento de arveja causado por Fusarium oxysporum. Corpoica. Ciencia y Tecnología Agropecuaria, 15(2), 237249.

Gutiérrez, L.A., y Zapata, J. (2011). Caracterización del agente causante de la Pudrición de raíces de la arveja (Pisum sativum L.), enfermedad endémica en el municipio de Manizales-Caldas (Colombia). Agronomía, 19(2): 33-43.

Helford, L.G., Stensvand, A., y Tronsmo, A. (2000). Effect of temperature and nutrient stress on the capacity of commercial Trichoderma products to control Botrytis cinerea and Mucor piriformis in greenhouse strawberries. Biological Control, 19, 149-160.

Keote, GA., Reddy, M.S.P., Kumar, B. M., y Wasnikar A.R. (2019). Bio-inoculants against chick pea wilt incited by Fusarium oxysporum f. sp. Cicero. Journal of Pharmacognosy and Phytochemistry, 8(4), 1590-1594. http://www.phytojournal.com/archives/2019/vol8issue4/PartAA/8-3-383-923.pdf

Kripalini, N., Kumar B.M., Devi S., y Sinha B. (2019). Studies on Survey of Fusarium wilt of Pea (Pisum sativum L.) and its Management by Native Trichoderma Isolates and Commercial Trichoderma under Pot Condition in Manipur. International Journal of Bio-resource and Stress Management, 10(1), 1-8. http://www.indianjournals.com/ijor.aspx?tar get $=$ ijor:ijbsm\&volume $=10 \&$ issue $=1 \&$ article $=001$

Leslie J.F., y Summerell B.A. (2006). The Fusarium Laboratory Manual. Blackwell Publishing, Hoboken, 1-2. https://doi.org/10.1002/9780470278376 Nagaraju U., Kumar, U., Harinikumar K.M., y Chavan M. (2017). Effect of bacterial isolates against Fusarium wilt in chick pea. Environment \& Ecology, 35(2A), 895-899. https://www. researchgate.net/profile/Mohan_Chavan3/publication/322138438_Effect_of_Bacterial_Isolates_Against_Fusarium_Wilt_in_Chick_Pea/ links/5a4768f40f7e9ba868aa9e98/Effect-ofBacterial-Isolates-Against-Fusarium-Wilt-inChick-Pea.pdf

Osorio, G., Castaño, Z., y Gutiérrez, R. (2012). In-vitro efficacy of plantain lixiviates on Fusarium oxysporum Schlecht, causal agent of pea (Pisum sativum Linneo) roots rot. Agronomía, 20(1), 17-25p.

Pabón J., y Castaño, J. (2012). Manejo de la pudrición radical de la arveja (Pisum sativum L) causada por Fusarium oxysporum Schlechtend. Agronomía, 20(2), 37-44, 2012. 
Peñaranda, G., y Molina, D.Y. (2011). La producción de arveja (Pisum sativum) en la vereda Monteadentro, provincia de Pamplona, Norte de Santander. Revista FACE, 8(11),43-56.

Tamayo, P.J. (2000). Enfermedades del cultivo de la arveja en Colombia: Guía de reconocimiento y control. Ministerio de Agricultura y Desarrollo Rural, Corpoica, Fenalce, Sena y SAC. p. 49.

Verma, D.K., Srivastava, S., Kumar, V. y Srivastav P.P. (2016). Biological Agents in Fusarium Wilt (FW) diagnostic for sustainable pigeon pea production, opportunities and challenges. In: Kumar P., Gupta V., Tiwari A., Kamle M. (eds) Current Trends in Plant Disease Diagnostics and Management Practices. p. 91-109. Fungal Biology Springer. https://link.springer.com/chapter/10.1007/978-3-319-27312-9_4

Viteri, S.E., Granados, M., y González, A.R. (2008). Potencial de los caldos rizosfera y súper cuatro como biofertilizantes para la sostenibilidad del cultivo de cebolla de bulbo (Allium cepa). Agronomía Colombiana, 26(3),517-524.

\section{(c) (i) (?)}

